\title{
Étude de l'engagement des apprenants dans un même cours en fonction de sa modalité : présentiel vs vidéo
}

\author{
CHRISTIAN HOFFMANN \\ Université Grenoble Alpes, CNRS, Grenoble INP, LIG ; Chercheur associé à l'EA ECP, \\ Univ. Lumière Lyon 2 \\ christian.hoffmann@univ-grenoble-alpes.fr \\ GILLES BUISSON \\ École des Ponts ParisTech, Service d'ingénierie et d'innovation pédagogique \\ gilles.buisson@enpc.fr
}

\section{Résumé}

Nous présentons dans cet article l'étude de l'engagement des étudiants d'une école d'ingénieur française dans un cours en fonction de sa modalité : cours en présentiel ou cours-vidéo. Sur la base de l'analyse de diverses données prises auprès d'étudiants sur deux ans, deux questionnaires écrits et 12 interviews, nous montrons que l'engagement est soutenu et indépendant de la modalité. Nous attribuons ce résultat entre autres aux caractéristiques des apprenants qui sont fortement motivés et possèdent une bonne connaissance de soi ; ce qui leur permet de choisir et de créer des conditions de travail adaptées afin de réussir leurs études. Finalement, nous examinons l'ensemble des spécificités de cette étude de cas afin d'extraire les conditions favorables à l'engagement des apprenants et concluons sur une possible transférabilité dans d'autres contextes.

\section{Summary}

We present the study of the engagement of students of a French engineering school in a course according to its modality: face-to-face or video-course. Based on the analysis of various data from these students over two years, two written questionnaires and 12 interviews, we show that the engagement is sustained, independent of the modality. Among other things, we attribute this 
to the characteristics of the learners who are highly motivated and have a good knowledge of themselves, which allows them to choose and create suitable working conditions in order to succeed in their studies. More generally, we examine all the specificities of the case studied in order to extract the conditions favorable to the engagement of the learners and conclude on a possible transferability in other contexts.

\section{Mots-clés}

Engagement académique, cours enregistré, école d'ingénieur

\section{Key words}

Academic commitment, recorded course, engineering school

\section{Introduction}

Le nombre de cours enregistrés proposés aux étudiants de l'enseignement supérieur, sous format vidéo et/ou diapositives sonorisées, croît significativement chaque année. Ces ressources ${ }^{1}$ sont destinées soit à remplacer les cours en présentiel, soit à les compléter. Si de nombreuses études, la plupart techno-centrées, évaluent l'impact de ces ressources sur les performances scolaires, on trouve moins d'études qui détaillent les habitudes de travail et les stratégies d'apprentissages d'étudiants lié à ce type de support. Roland (2012) relève la difficulté d'appréhender de manière efficace l'intégration du podcasting dans un dispositif pédagogique, tant leur « appropriation dans des stratégies d'apprentissage sont des processus riches, complexes, hétérogènes et répondant à des mécanismes conscients et réfléchis. ( $\mathrm{p}$. 307) ». Or, ces mécanismes constituent bien souvent des angles morts pour l'enseignant qui intègre la modalité vidéo à son cours, ce qui rend difficile d'accompagner les étudiants de façon optimale dans son utilisation. Comment expliciter ces modes d'appropriation de la vidéo par les étudiants ? Notre étude menée sur deux ans dans le cadre d'un cours scientifique à l'École des Ponts ParisTech cherche à mettre en évidence les effets de l'introduction d'un cours filmé en lieu et place d'un cours magistral traditionnel. Elle compare les deux modalités dans une

\footnotetext{
${ }^{1}$ Dans ce contexte, on parle souvent de podcasting (baladodiffusion), il s'agit d'un fichier vidéo/audio qui peut être téléchargé sur un ordinateur ou un support mobile. Dans le cas décrit ici, le téléchargement n'était a priori pas possible, nous utiliserons alors plutôt le terme « cours-vidéo » dans le texte.
} 
perspective exploratoire selon une méthode de recueil de données mixtes (quantitative et qualitative). Afin de contribuer à une approche systémique centrée sur les utilisateurs des dispositifs technologiques, comme proposée par Roland (2012), il s'agira, dans l'analyse du cas étudié, de faire ressortir les caractéristiques du public étudié et du dispositif dans son ensemble. Ceci permettra de pronostiquer en quoi des effets similaires pourraient être observés dans d'autres contextes.

Après avoir exposé la problématique, nous expliciterons le cadre de référence utilisé, à savoir le concept d'engagement. Puis, nous aborderons la méthodologie de l'étude, avant de présenter et de discuter les résultats obtenus.

\section{Contexte et problématique}

Les écoles d'ingénieur du réseau ParisTech expérimentent les cours enregistrés depuis plusieurs années déjà (Lemarchand et Olivier, 2009). Parmi elles, l'École des Ponts ParisTech incite les enseignants, dans le cadre d'une réforme de la formation d'ingénieur, à réduire le temps des cours en présentiel au profit du temps d'étude personnelle en autonomie pour les étudiants. Dans la perspective de ce changement, un professeur responsable d'un module de mécanique a mené une expérimentation : il a proposé aux étudiants de choisir entre suivre son cours en présentiel ou sous forme de vidéos, disponibles via une plateforme. Avec la mise en place de ces deux modalités en parallèle l'enseignant souhaitait récolter des éléments de réponse à un certain nombre d'interrogations qui le préoccupait : comment les étudiants gèrent leurs apprentissages quand on enlève les contraintes d'un cours en présentiel ? Est-ce que la formule vidéo comporte un risque de décrochage plus élevé que la modalité présentielle au point d'avantager un groupe par rapport à l'autre ? Est-ce que la vidéo est adaptée à un cours réputé exigeant sur le plan théorique?

Pour éclaircir ces liens entre l'introduction de la vidéo et les enjeux liés à l'autonomie dans l'apprentissage, nous mobilisons le concept d'engagement qui nous semble être un cadre théorique à la fois solide et propice pour appréhender les multiples dimensions en jeu pour l'étudiant à la fois cognitives, comportementales, mais aussi affectives. Nous formulons alors la question de recherche suivante : dans le cours de mécanique, comment le fait de suivre un cours en présentiel ou un cours enregistré au format vidéo influence l'engagement des apprenants? 
Avant de préciser comment nous caractérisons l'engagement dans cette étude, voici quelques détails du dispositif. L'enseignement en question est un module de deuxième année d'école (équivalent au niveau Master 1) et se compose essentiellement de cours magistraux (dix séances de 2 heures 30 ou 3 heures ${ }^{2}$ ), complétés par deux à quatre séances de «bureau d'étude » où les étudiants travaillent sur des cas, guidés par l'enseignant. Entre les séances de cours, les étudiants ont la possibilité de rendre des exercices (facultatifs) que l'enseignant corrige. Le professeur est très expérimenté dans le domaine de son cours qu'il donne depuis de nombreuses années et qu'il juge lui-même comme exigeant. Le support principal du cours est constitué des diapositives présentées pendant les séances magistrales. Ces diapositives, mises en ligne suite aux séances sur la plateforme pédagogique de l'école, tiennent presque lieu de polycopié, car elles détaillent chaque démonstration effectuée lors des séances. En complément, les étudiants disposent sur les quatre premières séances du cours d'un polycopié rédigé par l'enseignant.

Pour le cours-vidéo, les séances en présentiel d'une durée de 3 heures ont été enregistrées pendant l'année scolaire 2013-2014. Il s'agit donc de vidéos de cours « en temps réel ». La ressource se présente sous la forme d'une double fenêtre : une petite fenêtre vidéo où l'on voit le professeur qui donne son cours, assis derrière son bureau avec l'ordinateur ; une grande qui affiche les diapositives et où l'on peut observer le curseur de la souris permettant à l'enseignant de pointer des endroits spécifiques. L'agrandissement de la petite fenêtre peut être provoqué par l'étudiant à tout moment à l'aide d'un bouton. L'étudiant a également accès à un onglet qui récapitule les diapositives, ce qui lui permet d'aller directement à un endroit particulier du cours, puis à une fonction « recherche » où il peut chercher ce qui l'intéresse dans les mots clés qui ont été attribués à chaque diapositive.

Afin de comparer l'impact des deux formules, le professeur a proposé un choix aux étudiants : ils devaient décider en début d'année s'ils souhaitaient suivre le cours en présentiel ou sous forme de cours-vidéo. Ce choix était exclusif. A priori, les étudiants qui suivaient le cours en présentiel n'avaient pas accès au cours-vidéo, seulement aux diapositives et au polycopié. Les étudiants qui avaient choisi le groupe vidéo, avaient accès via la plateforme aux cours enregistrés (seulement pendant une dizaine de jours - la même semaine pendant laquelle se déroulait le cours en présentiel), au polycopié, aux diapositives et aux feuilles d'exercices.

\footnotetext{
${ }^{2}$ L'année 2 de notre étude, la durée du cours en présentiel a été réduite de $3 \mathrm{~h}$ à $2 \mathrm{~h} 30$.
} 


\section{Cadre de référence}

Le concept clé qui cadre notre analyse est l'engagement des étudiants dans un enseignement. Ce concept connaît un certain succès depuis les dernières décennies auprès des chercheurs et des praticiens dans les études de la réussite et du décrochage scolaire (Christenson et al., 2012). La littérature différencie entre la motivation et les formes de l'engagement liés à l'apprentissage académique avec des concepts comme le « commitment » (Kiesler, 1971, cité dans Pirot et De Ketele, 2000), le «student involvement » (Astin, 1984) ou encore le «student engagement » (Nystrand et Gamoran, 1991). Ces derniers distinguent l'engagement procédural, l'adhésion de l'étudiant aux règles et procédures de régulation de la classe et l'engagement substantif, l'attention soutenue portée aux contenus et aux visées de l'apprentissage.

Il y a aujourd'hui un consensus significatif parmi les chercheurs sur le fait que l'engagement est un concept multidimensionnel qui comporte des composantes comportementales et psychologiques (Maroco et al., 2016). Toutefois, il n’y a pas de consensus sur sa définition précise ou sur le nombre de dimensions.

Dans cette étude de cas, nous allons comparer les groupes présentiel et vidéo au regard des trois dimensions du concept d'engagement défini par Fredricks et al. (2004) : l'engagement comportemental, l'engagement émotionnel et l'engagement cognitif. L'engagement comportemental correspond à la conduite de l'étudiant en cours et plus généralement dans l'institution : présence en cours, remise des travaux demandés, degré d'implication dans les tâches d'apprentissage académiques et dans des activités liées à l'école comme les associations étudiantes. L'engagement émotionnel comprend les réactions positives et négatives envers l'enseignant, les camarades de classes, l'environnement académique et l'école. L'engagement cognitif, étudié par exemple par Miller et al. (1996), se rapproche de l'engagement substantif de Nystrand et Gamoran (1991). Il croise l'approche de la littérature sur l'implication dans l'apprentissage et celle sur les stratégies d'apprentissages (Bégin, 2008) et l'apprentissage autorégulé (Cosnefroy, 2011 ; Houart, 2017). Les indicateurs sont par exemple l'investissement que l'étudiant déploie pour comprendre les idées complexes et maitriser les habiletés difficiles, la résistance contre les distractions, les stratégies pour s'approprier le cours et réviser, la capacité à tenir compte de ses erreurs pour modifier ses stratégies d'apprentissage (métacognition). 


\section{Méthodologie}

\subsection{Sujets}

Il y a respectivement 45 étudiants qui ont assisté au cours pendant l'année 2014/2015 et 39 pendant l'année 2015/2016. L'année 1, assez peu d'étudiants se sont proposés spontanément pour le groupe vidéo, mais suite à quelques encouragements de l'enseignant, 18 personnes ont choisi le groupe vidéo et 2, le groupe présentiel. L'année 2, les groupes ont été plus inégaux : 7 étudiants pour le groupe vidéo et 32 pour le groupe présentiel.

Au niveau du parcours académique antérieur des étudiants, il faut noter trois types de publics distincts : les étudiants qui ont suivi la première année de la formation d'ingénieur aux Ponts ; les étudiants qui intègrent l'École des Ponts en complément de leurs parcours de formation d'ingénieur, notamment des étudiants de Polytechnique ou de l’École normale supérieure ; les étudiants étrangers en double diplôme.

Pour situer notre étude de cas, il est important de garder en tête que le public n'est pas représentatif de la majorité des étudiants / étudiantes au niveau de l'enseignement supérieur car l'école est très sélective et réunit, sauf exception, des étudiants avec un très solide bagage scientifique, une grande autonomie et un projet professionnel bien défini. Nous reviendrons sur les caractéristiques de ce public dans la discussion des résultats.

\subsection{Instrumentation / Collecte de données}

Année 1 : Pour étudier l'engagement des apprenants, mais également d'autres aspects du dispositif (non exploités ici), nous avons conçu deux questionnaires sur mesure pour interroger les étudiants qui suivaient le module : les deux étaient à remplir en ligne sur la plateforme pédagogique.

Une première enquête a été soumise à mi-parcours fin novembre. Cette enquête était courte (cinq champs à renseigner) et exclusivement sous forme de questions ouvertes. Les étudiants ont été interrogés sur les motifs de leur choix, les avantages et risques qu'ils voyaient pour la modalité choisie et leur satisfaction avec leur choix à ce stade. En fin de questionnaire, il y avait un champ pour des remarques/commentaires libres. Le taux de réponse a été de $100 \%$ dans les deux groupes. 
Une deuxième enquête leur a été soumise en fin d'enseignement (après l'examen). Le questionnaire du groupe présentiel comporte 11 questions fermées, dont 8 évaluées sur une échelle de Likert de quatre niveaux (concernant, par exemple, l'attention en cours, l'utilisation des supports mis à disposition, la satisfaction avec le choix de la modalité) et 3 questions oui/non, accompagnées de champs de commentaires (concernant entre autres l'évolution de l'implication dans le module, les changements souhaités pour le module); 4 questions quantitatives (par exemple sur le temps de travail) et une question ouverte (points forts du module). En plus de ces mêmes questions, les étudiants du groupe vidéo ont été interrogés sur leurs habitudes de visionnage et la qualité des ressources. Le taux de réponses a été de $81 \%$ (22/27) pour le groupe présentiel et de $83 \%(15 / 18)$ pour le groupe vidéo.

Année 2 : La deuxième année nous avons souhaité approfondir certains questionnements à travers une série d'entretiens semi-directifs avec des étudiants des deux modalités. La volonté a été de constituer deux groupes équitables pour chaque modalité. On a ainsi pu dialoguer avec sept étudiants ayant suivi le cours en présentiel et cinq étudiants ayant suivi le cours en vidéo sur des durées allant de 30 à 45 minutes. La première partie de la grille d'entretien créée pour l'occasion, portait sur la perception de la cohérence pédagogique du cours à travers des questions sur les trois piliers de l'alignement pédagogique, à savoir les objectifs, les activités et l'évaluation du module (pas exploitée ici). La deuxième partie s'intéressait plus en détail aux stratégies d'apprentissage déployées par les étudiants aux différentes étapes du dispositif : pendant le cours magistral ou le visionnage des vidéos, pendant les bureaux d'études, pendant le travail de révision ou les devoirs à rendre à la maison, etc. Chaque entretien a été ensuite retranscrit et anonymisé. Ainsi, le code retenu pour chaque étudiant va d'E1 à E12.

\subsection{Indicateurs de l'engagement}

Le Tableau 1 ci-dessous présente les indicateurs retenus dans notre étude pour chaque dimension de l'engagement. 


\begin{tabular}{|l|l|l|}
\hline Engagement émotionnel & $\begin{array}{l}\text { Engagement } \\
\text { comportemental }\end{array}$ & Engagement cognitif \\
\hline - Motifs initiaux pour le \\
choix de la modalité, \\
$\begin{array}{l}\text { avantages et risques } \\
\text { perçus }\end{array}$ & $\begin{array}{l}\text { Régularité dans la } \\
\text { présence en cours / } \\
\text { dans le visionnage des } \\
\text { vidéos }\end{array}$ & $\begin{array}{l}\text { Attention en cours / } \\
\text { lors des visionnages } \\
\text { (stratégies de } \\
\text { visionnage) }\end{array}$ \\
$\begin{array}{l}\text { Satisfaction a } \\
\text { posteriori par rapport } \\
\text { aux modalités choisies }\end{array}$ & $\begin{array}{l}\text { l'enseignant et les pairs } \\
\text { Temps de travail } \\
\text { personnel global }\end{array}$ & $\begin{array}{l}\text { fombre d'exercices } \\
\text { Appropriation du cours } \\
\text { (prise de notes et leur } \\
\text { reprise après) }\end{array}$ \\
\hline
\end{tabular}

Dans la littérature, il n'y a pas de consensus sur le nombre de dimensions de l'engagement et sur leurs frontières (Maroco et al., 2016). De ce fait, il est difficile de classer certains indicateurs, par exemple, le temps global de travail. Nous avons fait le choix de le situer dans la dimension comportementale plutôt que cognitive, car le temps passé n'est pas impérativement équivalent à un temps d'engagement cognitif fort. Ceci rejoint la différence que la littérature anglophone fait entre Allocated Time, Engaged Time et Active Learning Time (Gettinger et Seibert, 2002). Pour le nombre d'exercices rendus, nous avons fait le choix inverse du fait de leur caractère facultatif. En dépit de ces problèmes, étudier conjointement les trois dimensions permet de capter l'essentiel du concept.

\section{Résultats}

\subsection{Engagement émotionnel}

\section{Motifs initiaux pour le choix de la modalité, avantages et risques perçus}

Si le choix de la modalité a été laissé à l'appréciation des étudiants pour les deux ans, il n'était spontané que pour une petite partie d'entre eux. En effet, l'année 1, le professeur a incité certains étudiants à choisir la modalité vidéo afin de constituer des groupes à peu près équivalents en taille. L'année 2, le choix vidéo n'étant pas particulièrement encouragé, seulement sept étudiants l'ont fait dont deux contraints par leur emploi du temps.

Interrogés à mi-parcours du cours lors de l'année 1 sur les avantages de la modalité vidéo, les étudiants évoquent la flexibilité dans l'organisation de leur emploi du temps. Les entretiens de 
l'année 2 confirment, pour l'ensemble des étudiants interrogés, l'intérêt de la souplesse offerte par la vidéo pour organiser son travail à son rythme. Cette flexibilité relève d'abord de la possibilité de partitionner le visionnage de la vidéo en un ou plusieurs créneaux horaires choisis dans la semaine et aussi dans la possibilité d'adapter le rythme du discours de l'enseignant en fonction de la complexité du passage. Le deuxième motif qui ressort dans l'enquête de l'année 1 pour le choix de la vidéo est l'envie de découvrir ce média. Dans l'entretien E8, l'étudiant avait déjà vécu l'expérience réussie d'un cours en vidéo et voulait la reconduire. Les risques évoqués par le groupe vidéo sont la possibilité d'accumulation de retard dans le visionnage ; de regarder le cours de façon distraite ; d'être moins captivé par le professeur ou encore de passer trop de temps sur une vidéo.

Par contraste, le choix initial du présentiel pour les étudiants de l'autre groupe a été motivé, selon l'enquête de l'année 1 et les entretiens de l'année 2, par le fait de préférer avoir une contrainte horaire pour le cours et la crainte de ne pas être en capacité de s'astreindre à regarder la vidéo du cours filmé à la maison. L'autre motif avancé est l'impression d'être plus attentif dans une salle de classe qu'à la maison devant un écran. Enfin, les étudiants évoquent la possibilité de poser des questions au professeur en séance. Le risque principal que les étudiants mentionnent fréquemment est de décrocher du discours car «c'est le professeur qui décide du rythme adopté ». Le point faible qui ressort est la durée du cours, deux heures et demies d'affilée.

\section{Satisfaction a posteriori par rapport aux modalités choisies}

Sur la satisfaction du choix de la modalité a posteriori pour l'année 1, nous avons interrogé les deux groupes à l'aide des 3 items suivants :

I. Le choix du mode « $\mathrm{xxx} » \mathrm{~m}$ 'a permis de mieux organiser mon travail pour cette matière et de le rendre plus efficace.

II. Je pense que j'aurais pu obtenir de meilleurs apprentissages en suivant le cours en « Xxx ». (l'autre modalité)

III. Avec du recul, à la fin du module, je suis content de mon choix pour la modalité « XXX ». 
Les résultats pour ces 3 questions sont résumés dans le tableau 2 ci-dessous :

\begin{tabular}{|l|l|l|l|l|l|l|}
\hline Question & \multicolumn{2}{l}{ I. II. } & \multicolumn{2}{l|}{ III. } \\
\hline Groupe & Présentiel & Vidéo & Présentiel & Vidéo & Présentiel & Vidéo \\
\hline $\begin{array}{l}\text { Tout à fait } \\
\text { d'accord }\end{array}$ & 12 & 3 & 0 & 1 & 14 & 8 \\
\hline Plutôt d'accord & 7 & 8 & 3 & 2 & 5 & 5 \\
\hline $\begin{array}{l}\text { Plutôt pas } \\
\text { d'accord }\end{array}$ & 2 & 2 & 9 & 4 & 3 & 1 \\
\hline $\begin{array}{l}\text { Pas du tout } \\
\text { d'accord }\end{array}$ & 0 & 1 & 9 & 5 & 0 & 1 \\
\hline Sans opinion & 1 & 1 & 1 & 3 & 0 & 0 \\
\hline
\end{tabular}

Globalement, on constate qu'a posteriori seulement 2 ou 3 étudiants dans chaque groupe ne sont pas satisfaits de leur choix et pensent qu'ils auraient pu obtenir de meilleurs apprentissages avec l'autre modalité. Le seul étudiant qui répond «pas du tout d'accord » aux questions I. et III. s'est rendu compte rapidement que son choix initial ne lui convenait pas - il l'avait déjà indiqué dans l'enquête de mi-parcours de l'année 1. Les entretiens de l'année 2 confirment la satisfaction du choix de la modalité pour l'ensemble des étudiants interrogés.

Pour compléter les indicateurs de l'engagement émotionnel, notons également la présence de deux éléments contextuels au cours qui agissent sur cette dimension. Le cours a la particularité d'être plus exigeant sur le plan mathématique que des cours similaires dans d'autres écoles. En plus d'être un des cours les plus importants de la deuxième année au niveau du département, il est décrit par le professeur et par les apprenants comme un vrai défi intellectuel. Les étudiants soulignent dans les entretiens les qualités de pédagogue de l'enseignant qui fait par ailleurs figure d'autorité dans son domaine à l'École. 


\subsection{Engagement comportemental}

\section{Régularité dans la présence en cours / dans le visionnage des vidéos}

Pour les deux années, il n'y a quasiment pas d'absentéisme dans le cours en présentiel. Le questionnaire de l'année 1 révèle que 13 étudiants sur les 15 répondants du groupe vidéo ont visionné toutes les vidéos. Les deux étudiants qui n'ont pas tout regardé déclarent avoir vu respectivement $75 \%$ et $50 \%$ des vidéos. Pour l'année 2, tous les étudiants, sauf un, ont visionné toutes les vidéos.

\section{Interactions avec l'enseignant et les pairs}

Des différences notables entre les deux groupes apparaissent au niveau des interactions entre étudiants et avec le professeur en dehors du cours. Lors de l'année 1, dans les deux groupes, environ $40 \%$ des répondants ne travaillent jamais ou peu avec les autres étudiants $(<10$ heures sur la totalité de l'enseignement), mais pour les 60 \% restant, le temps moyen qu'ils ont passé à travailler avec leurs camarades est bien plus élevé dans le groupe présentiel (33 heures, contre 19 heures pour le groupe vidéo). Concernant l'interaction avec le professeur : seulement deux étudiants du groupe vidéo ont posé au moins une question au professeur par mail et également deux l'ont rencontré pour lui poser des questions ; pour comparaison, dans le groupe présentiel, ils sont huit et douze respectivement. L'année 2, deux étudiants sur cinq interrogés pour la modalité vidéo ont sollicité l'enseignant contre trois sur sept pour le présentiel.

\section{Temps de travail personnel global}

L'année 1, nous avons interrogé les étudiants concernant le temps global qu'ils ont investi sur ce module. Pour le groupe présentiel, le temps de travail personnel en dehors du cours s'inscrit dans une fourchette entre 15 et 70 heures avec une moyenne à 40 heures. La question similaire pour le groupe vidéo n'est malheureusement que peu exploitable car certains étudiants semblent avoir mal lu l'énoncé. Deux étudiants indiquent 20 heures à la question : «Combien de temps avez-vous travaillé pour ce module, y compris le temps passé à visionner les vidéos ? Indiquez un nombre d'heures », en contradiction avec leur affirmation par ailleurs d'avoir visionné toutes les vidéos dans un temps au moins équivalent à la durée des vidéos (25 à 30 heures au total pour les 10 séances). La moyenne obtenue pour le groupe vidéo, à savoir 56 heures, c'est-àdire une trentaine d'heures en plus de la durée des vidéos, est donc certainement sous-estimée. Ceci pris en compte, on peut extrapoler que le temps de travail personnel moyen pour ce module 
n'est pas très différent pour les deux groupes. En revanche, on constate une forte variation entre étudiants. Pour le groupe vidéo, la fourchette (visionnage inclus) est de 20 à 80 heures.

\subsection{Engagement cognitif}

\section{Attention en cours / lors des visionnages (stratégies de visionnage)}

Dans l'enquête de l'année 1, 4 sur 22 répondants admettent des difficultés à mobiliser leur attention lors des cours en présentiel. En revanche, il n'y a qu'un seul étudiant sur 15 répondants qui le déclare pour les vidéos. Les stratégies de visionnage sont très variées : huit étudiants ont réservé une ou plusieurs plages horaires fixes dans la semaine, les autres changeaient le créneau de semaine en semaine en fonction de leurs disponibilités. Un tiers des étudiants visionnait les cours en une fois, deux tiers le répartissaient sur plusieurs créneaux. Ces données « autodéclarées » sont corroborées par les statistiques de connexion sur la plateforme. En ce qui concerne le temps total passé pour visionner un cours de trois heures, cinq étudiants le visionnent sans grandes interruptions (donc en 3 heures environ) les 10 autres prennent plus de temps.

L'année 2, en présentiel, à l'exception de l'étudiant E9 qui déclare ne pas avoir eu de difficulté à suivre les séances de cours magistral, les autres étudiants avouent avoir décroché à certaines séances aux passages compliqués ou calculatoires de l'exposé. Dans les entretiens du groupe vidéo de l'année 2, deux étudiants déclarent avoir visionné les vidéos en une fois (E8 et E12) et deux en plusieurs fois (E2 et E10). Deux étudiants affirment avoir visionné les vidéos à un créneau fixe de la semaine (E10 et E12) et la plupart le faisait aussi le week-end (E8, E10, E11, E12). L'ensemble des étudiants ont visionné les vidéos avec des pauses, certains en accéléré ou avec des retours en arrière (E8 et E10). Sur l'ensemble des étudiants du groupe vidéo, on note de grandes disparités sur le temps de visionnage dû à des niveaux hétérogènes sur la matière ou sur la langue. Ainsi, l'étudiant E2 estime avoir passé jusqu'à dix heures pour visionner une vidéo, car d'origine chinoise, il lui fallait plus de temps pour comprendre le cours que pour l'étudiant E11 ayant déjà quatre ans de mécanique derrière lui et qui n'a plus visionné les vidéos après la cinquième séance se reposant uniquement sur les diapositives de l'enseignant.

\section{Nombre d'exercices facultatifs rendus}

Pendant l'année 1, le groupe vidéo déclare avoir rendu un peu plus régulièrement des exercices (73\% versus $64 \%$ pour le groupe en présentiel). La tendance est la même 
l'année 2 avec trois étudiants sur cinq qui ont rendu plus d'un exercice facultatif. En comparant cette donnée sur la base d'un fichier de l'enseignant qui inclut la totalité de l'effectif de l'année 2, on ne constate pas de différence significative entre le groupe présentiel et le groupe vidéo.

\section{Appropriation du cours (prise de notes et leur reprise après)}

Concernant les stratégies de prise de notes, les proportions des personnes qui prennent des notes en regardant le cours sont comparables l'année 1:63\% pour le présentiel, $60 \%$ pour la vidéo. L'utilisation des supports de cours est également similaire dans les deux groupes : seulement $40 \%$ ont régulièrement étudié le polycopié (36\% pour le présentiel, $40 \%$ pour la vidéo) mais quasiment $100 \%$ ont régulièrement consulté les diapositives du cours sur la plateforme (100\% pour le présentiel, $93 \%$ pour la vidéo). Les similarités se poursuivent l'année 2 avec des stratégies variées en vidéo comme en présentiel. En vidéo, trois étudiants prennent des notes (E2, E10, E12) essentiellement pour refaire les démonstrations les plus difficiles, les deux autres n'en sentent pas le besoin. En présentiel, certains annotent les diapositives sur ordinateur, d'autres préfèrent ne pas avoir l'ordinateur devant eux mais de porter toute leur attention sur le discours du professeur. Les étudiants qui déclarent décrocher " par moment », indiquent qu'ils rattrapaient leur retard grâce au support de cours et au travail de relecture et de révision à la maison.

\subsection{Lien entre les résultats au module et la modalité choisie}

Pour compléter les données récoltées, quelques remarques sur les notes obtenues par les étudiants dans ce module. Pour les deux années, la réussite globale dans le module est très bonne. Année $1: 38$ étudiants sur 45 obtiennent une note entre 18 et 20 sur 20 ; Année $2: 25$ étudiants sur 39 obtiennent une note entre 16 et 20. Aucun étudiant n'a pas validé le module. Pas de différence évidente entre les deux groupes, vidéo et présentiel. Les quelques étudiants avec des notes moins bonnes (moins de 4 étudiants par an avec des notes inférieures à 14) sont des cas particuliers : étudiants étrangers qui sont encore en phase d'adaptation dans cette école et un ou deux décrocheurs.

Ces résultats s'expliquent d'une part par la «philosophie » de notation du professeur qui « ne souhaite pas que les étudiants trouvent leur motivation dans la crainte de ne pas valider ce module. ». D'autre part, le système de notation permet d'obtenir de nombreux points et demi, 
points de bonus pour un étudiant qui accepte de faire le travail facultatif. La différence entre l'année 1 et 2 (moyenne inférieure) est due à la difficulté de l'examen final, d'habitude très exigeant. Lors de la première année d'expérimentation, il était plus facile que les années précédentes et l'année suivante, car l'enseignant craignait de désavantager les étudiants du groupe vidéo.

\subsection{Remarque globale}

Les différences dans les parcours académiques antérieurs des étudiants ne sont pas discriminantes sur les dimensions cognitives et comportementales de l'engagement, à l'exception du temps de visionnage pour les étudiants avec des difficultés de langue. Elles expliquent cependant une partie des choix initiaux de la modalité vidéo car ce sont essentiellement les étudiants qui intègrent l'École des Ponts en complément de leurs parcours de formation d'ingénieur qui choisissent par contrainte de leur emploi du temps la modalité vidéo en année 2.

\section{Discussion}

Les résultats nous amènent globalement à constater que pour les sujets étudiés, l'engagement dans le cours est fort, à quelques exceptions près et indépendamment de la modalité choisie. Examinons maintenant les 3 dimensions de plus près.

Du point de vue de l'engagement émotionnel, on aurait pu penser que le support audiovisuel qui tranche avec le mode traditionnel d'enseignement (cours magistral en présentiel) allait motiver les étudiants. Force est de constater que peu d'entre eux l'ont choisi spontanément, au contraire, beaucoup d'étudiants ont une appréhension a priori vis-à-vis du cours vidéo. L'analyse de l'indicateur «motifs initiaux pour le choix » nous montre que l'appropriation de la vidéo dépend de la confiance des étudiants dans leur capacité à adapter leurs habitudes de travail à un support qui introduit un rapport au temps et à l'espace différent du présentiel. Les étudiants de l'école des Ponts ParisTech ont très bien réussi leur scolarité dans un système traditionnel (cours en présentiel, fortement encadrés en classes préparatoires et à l'école) et ils ont conscience que la vidéo demande plus d'autorégulation et de nouvelles stratégies d'apprentissage. Alors seulement lorsque les étudiants se sentent capables de répondre à ces exigences, on arrive à une attitude qui valorise les propriétés intrinsèques de la vidéo comme support d'apprentissage. Ils utilisent par exemple les fonctions stop, pause, rewind et ils 
s'attardent sur des points qui les intéressent et les enrichissent avec de nouvelles sources, des effets également observés par Roland et Emplit (2015). Par conséquent, ils peuvent aboutir à des stratégies d'élaboration de supports de révision qu'ils n'auraient pas employé en présentiel. Le taux de satisfaction a posteriori nous montre que peu d'étudiants regrettent à la fin leur choix, même s'il était un peu contraint au départ pour certains membres du groupe vidéo. Au contraire, avoir ce choix leur a permis de mieux organiser leur travail pour ce module et de le rendre plus efficace (affirmation par 19 répondants sur 22 dans le groupe présentiel et 11 sur 15 pour le groupe vidéo, cf. tableau 2). Cela indique que le public étudié, étudiants-ingénieur des grandes écoles, a dans la majorité recours à des réflexions métacognitives qui les conduisent à éviter des risques dans le choix de modalités d'apprentissage nouvelles, mais qui leur permettent également de s'y adapter consciemment s'ils sont obligés.

Concernant l'engagement comportemental, le choix de la modalité ne semble pas critique, en témoigne la régularité de la présence en cours et le taux de visionnage. Une fois que l'étudiant s'engage donc dans une modalité ou l'autre, il fait le nécessaire pour valider le module, si ce n'est pour quelques rares exceptions, a minima. On peut noter que le présentiel engage plus les étudiants à aller consulter l'enseignant ou ses camarades en dehors de la classe que ne le fait le cours filmé, même si les profils individuels des étudiants pourraient amener à nuancer ce constat. Un étudiant suivant le cours en présentiel aura alors plus de facilité à établir des liens sociaux pouvant l'aider dans le cas où il rencontre plus de difficultés qu'un étudiant du groupe vidéo. En ce sens, l'enseignant a exprimé à la fin de l'expérimentation, l'intention d'orienter les étudiants en difficulté vers le cours en présentiel. Nous pouvons cependant dire que leur donner en plus accès à la ressource vidéo sera certainement un atout pour eux, notamment pour revoir les passages difficiles du cours ou pour les étudiants étrangers ayant des difficultés linguistiques.

Pour la dimension de l'engagement cognitif, il est intéressant de s'attarder sur l'évaluation du risque de décrochage. Les résultats nous indiquent qu'un décrochage attentionnel «sur le moment » peut se produire dans les deux modalités, mais il est de nature différente et les « remèdes » le sont également. En présentiel, un décrochage peut se produire à cause du rythme imposé par le professeur, un étudiant en difficulté ne peut pas profiter d'une pause pour assimiler un concept important pour la suite de la séance et perd le fil. Il peut certes interrompre le professeur pour poser une question ou aller le voir en fin de cours pour avoir les éléments qui lui permettront de « raccrocher le train », mais pour certains cela ne sera pas suffisant : « en 
théorie c'est mieux parce qu'on peut poser des questions à l'enseignant. Mais quand on est perdu dès le début parce que le cours s'enchaîne trop vite, on n'a pas le temps de se poser les bonnes questions...» (extrait d'une réponse à une question ouverte d'un questionnaire de l'année 1$)^{3}$. Malgré tout, les étudiants qui ont choisi la modalité présentiel préfèrent retravailler a posteriori les parties où ils ont décroché en s'appuyant sur leur mémoire et/ou sur leurs notes, même incomplètes, plutôt que de prendre le risque de ne pas savoir gérer le visionnage des vidéos (manque d'autodiscipline, etc.). Dans le cas du cours vidéo, c'est plutôt le manque de contact avec le professeur qui peut poser souci et conduire au décrochage. Une incompréhension ou une petite imprécision dans le cours, qui se corrige facilement en présentiel, peut ici devenir un obstacle sur lequel l'étudiant butte à la maison. D'autre part, il peut prendre du retard dans le visionnage des vidéos, s'il n'arrive pas à bien s'organiser. Dans les deux modalités, un décrochage « global » (on entend par là qu'au fil des semaines, l'étudiant n'arrive plus à rester à jour concernant l'assimilation des connaissances du cours) peut être la conséquence de décrochages «sur le moment » répétés, si l'étudiant ne réagit pas de façon adéquate. Les résultats de notre étude montrent que ce genre de décrochage « global» reste cependant une exception pour le public de ce cours. Ceci s'explique par une forte motivation intrinsèque à ce stade de leur parcours de formation, car il s'agit d'étudiants de deuxième année qui ont pu choisir en fin de première année leur département de spécialisation.

D'un point de vue plus global, notons qu'il est difficile de tirer des conclusions concernant une corrélation entre la modalité et la réussite au module pour des raisons que nous avons évoquées en section 5.4. Ceci dit, nous n'avons pas observé de différence dans la distribution des notes entre les deux groupes, vidéo et présentiel. En revanche, nous avons pu mettre en évidence que, malgré une forte homogénéité concernant leurs pré-acquis et leurs parcours scolaires antérieurs (à quelques exceptions près, ils sont tous issus de classes préparatoires, puis d'une école d'ingénieur), les habitudes de travail et les préférences en termes de support à l'apprentissage varient fortement. Certains étudiants préfèrent les contraintes et l'ambiance de travail d'un cours en présentiel, d'autres la liberté de s'organiser à leur guise pour suivre le cours. Les uns aiment travailler seul, d'autres ont travaillé jusqu'à 50 heures avec leurs camarades ou questionnent fréquemment le professeur. Dans les deux groupes, on trouve des étudiants disant que leur attention portée au cours est bien meilleure dans la modalité choisie que s'ils étaient

\footnotetext{
${ }^{3}$ Il est intéressant de noter que peu d'étudiants ont décrit des problèmes d'attention en présentiel dans le questionnaire alors que presque tous l'avouent lorsqu'on approfondit le questionnement dans les interviews.
} 
obligés de suivre l'autre et environ $60 \%$ des étudiants prennent des notes pendant le cours, indépendamment de la modalité.

Cette forte variabilité dans les habitudes et le choix des modalités de travail pourraient tout aussi bien être observés chez des publics plus hétérogènes au regard de leur parcours antérieur et de leurs pré-acquis, comme on les rencontre par exemple à l'université. Mais qu'en est-il de l'apprentissage avec un cours vidéo ? Afin d'établir des hypothèses qui pourraient être mises à l'épreuve dans d'autres études, nous résumons ici les caractéristiques du cas étudié. Sauf exception, on peut supposer que les sujets dans cet établissement très sélectif se distinguent par une très bonne maîtrise des prérequis, une forte perception de compétence, une forte motivation intrinsèque, des méthodes de travail efficaces et souvent un projet professionnel bien identifié. Tous ces facteurs contribuent à un engagement soutenu, comme identifié par de nombreuses recherches (Pirot et De Ketele, 2000). D'autre part, le cours représente un défi auquel les étudiants sont sensibles et ils savent que l'enseignant, qui est souvent présent à l'École, est disponible pour des échanges individuels. Enfin, l'entraide entre pairs est fréquente. Ces facteurs, combinés avec une grande capacité de travail, permettent aux étudiants de pallier seul ou avec leurs pairs les faiblesses des deux modalités proposées et de surmonter des difficultés dans l'apprentissage. Par contraste, en début de cycle à l'université, ces facteurs ne sont pas souvent réunis. Les risques de décrochage «sur le moment» et «global», comme décrits supra, sont plus élevés avec des conséquences négatives pour l'engagement dans les cours et la réussite académique.

Parmi les points intéressants qui ressortent de notre étude sur l'appropriation du podcast et qui peuvent être généralisés, notons d'abord le poids de l'affectif dans le choix initial du dispositif. L'attirance du public étudiant pour les podcasts qu'on peut observer fréquemment dans le cadre privé, n'est pas transposable dans le contexte des études. S'agissant des habitudes de travail, l'apprenant ne sort pas facilement des sentiers battus, encore moins s'ils l'ont amené à la réussite jusqu'ici. Deuxièmement, face à un contenu exigeant sur le plan théorique, l'attention est plus facile à maintenir devant un cours vidéo. En effet, les étudiants ont toujours la possibilité de faire une pause afin d'assimiler à leur rythme les étapes d'une démonstration ou de croiser différentes sources d'information. Pour autant, l'étude solitaire devant un écran a aussi des inconvénients. Par exemple, l'apprenant ne peut pas poser de questions au sujet d'une erreur dans les diapositives qui peut alors devenir un point bloquant. Le manque de proximité avec le professeur et les pairs peut aussi nuire à l'intégration sociale de l'étudiant dans 
l'établissement si beaucoup de cours sont dispensés sous cette forme, avec de possibles conséquences pour les résultats académiques. En effet, Tinto a montré l'importance de l'intégration sociale en début de cycle universitaire pour l'intégration et la réussite académique (Tinto, 1997).

\section{Conclusion de l'étude}

Cette étude de cas nous a permis d'apporter un éclairage sur les effets de l'introduction d'un cours filmé en lieu et place d'un cours magistral sur l'engagement des étudiants. Nous donnons des éléments de réponse à des questionnements qui se posent de manière générale dans le contexte universitaire, mais dans le cadre d'un dispositif pédagogique précis avec un public qui a ses propres caractéristiques, les étudiants d'une grande école. L'analyse des avis des apprenants et du contexte nous amène à la conclusion que laisser le choix de la modalité du cours permet aux étudiants une utilisation efficace des ressources en accord avec leurs préférences de travail, que nous avons décrit en détail. Pour le public concerné ici, le choix initial de la modalité a été quasiment toujours satisfaisant, car il se base sur une bonne connaissance de soi. Pour les étudiants les plus en difficulté, avoir accès aux vidéos en plus du cours en présentiel peut être une aide appréciable, mais basculer entièrement le cours sous forme enregistrée pourrait mettre en difficulté les étudiants qui préfèrent largement le cours en présentiel. Nous avons effectivement pu observer une grande variabilité entre les étudiants dans la façon de s'approprier le cours et d'interagir avec l'enseignant et les pairs. Nous pouvons cependant supposer qu'au regard de leurs pré-acquis et leur forte capacité de travail, les étudiants réussiront malgré tout. Rappelons que les caractéristiques de l'échantillon étudié ici sont non généralisables à la majorité du public dans l'enseignement supérieur français. Il serait intéressant de reproduire une étude semblable sur un échantillon plus large, par exemple en Licence ou à l'Institut Universitaire de Technologie (IUT). Si la tendance actuelle est effectivement de proposer une plus grande flexibilité dans les cursus pour ces publics, il serait bénéfique de prévoir un accompagnement tout au long des dispositifs. On pourrait alors aider les étudiants à adopter plus systématiquement une posture métacognitive, par exemple questionner les préconceptions et les expériences antérieures afin de faire des choix pertinents et ainsi prévenir les risques de décrochage aux différents niveaux que nous avons identifiés. Cet accompagnement méthodologique est d'autant plus nécessaire lorsqu'on impose une modalité unique du cours, par exemple le cours filmé qui, pour une partie des étudiants, ne convient pas à leurs habitudes et préférences de travail. 


\section{Références bibliographiques}

Astin, W.A. (1984). Student involvement: A developmental theory for higher education. An empirical typology of college students. Journal of College Student Personnel, 25, 297-308.

Bégin, C. (2008). Les stratégies d'apprentissage : un cadre de référence simplifié. Revue des sciences de l'éducation, 34(1), 47-67.

Christenson, S.L., Reschly, A.L. et Wylie, C. (2012). Handbook of research on student engagement. New York: Springer Science + Business Media.

Cosnefroy, L. (2011). L'apprentissage autorégulé, entre cognition et motivation. Grenoble : PUG.

Fredricks, J., Blumenfeld, P. et Paris, A. (2004). School Engagement: Potential of the Concept, State of the Evidence. Review of Educational Research, 74(1), 59-109.

Gettinger, M. et Seibert, J.K. (2002). Best practices in increasing academic learning time. Dans A. Thomas et J. Grimes (dir.), Best practices in school psychology IV (p. 773-787). Bethesda: National Association of School Psychologists.

Houart, M. (2017). L'apprentissage autorégulé : quand la métacognition orchestre motivation, volition et cognition. Revue internationale de pédagogie de l'enseignement supérieur, 33(2). https://doi.org/10.4000/ripes.1246

Lemarchand, S. et Olivier, K. (2009). Enregistrement de cours en vue de leur baladodiffusion. Avis et retours d'expérience des étudiants, des enseignants et des internautes. Distances et Savoirs « TICE, enseignement supérieur et formation », 7(2), 205-217.

Maroco, J., Maroco, A.-L., Alvares Duarte Bonini Camos, J. et Fredricks, J. (2016). University student's engagement: development of the University Student Engagement Inventory (USEI) Psicologia: Reflexão e Crítica, 29(21). doi : 10.1186/s41155-016-0042-8.

Miller, R.B., Greene, B.A., Montalvo, G.P., Ravindran, B., Nichols, J.D. (1996). Engagement in Academic Work: The Role of Learning Goals, Future Consequences, Pleasing Others, and Perceived Ability. Contemporary Educational Psychology, 21, 388-422.

Nystrand, M. et Gamoran, A. (1991). Instructional discourse, student engagement, and literature achievement. Research in the Teaching of English, 25(3), 261-290.

Pirot, L. et De Ketele, J.-M. (2000). L'engagement académique de l'étudiant comme facteur de réussite à l'université Étude exploratoire menée dans deux facultés contrastées. Revue des sciences de l'éducation, 26(2), 367-394.

Roland, N. (2012). Intégration du podcasting à l'université : pourquoi ? Comment? Pour quels résultats? Actes du $27^{\mathrm{e}}$ Congrès de l'Association internationale de pédagogie universitaire (AIPU), Trois-Rivières, Canada, 301-308.

https://oraprdnt.uqtr.uquebec.ca/pls/public/gscw031?owa_no_site $=2220$

Roland, N. et Emplit, P. (2015). Enseignement transmissif, apprentissage actif : usages du podcasting par les étudiants universitaires. Revue internationale de pédagogie de l'enseignement supérieur, 31(1). https://doi.org/10.4000/ripes.932

Tinto, V. (1997). Classrooms as Communities: Exploring the Educational Character of Student Persistence. Journal of Higher Education, 68(6), 599-623. 


\section{Remerciements}

Nous remercions J.-Y. Poitrat de l'École des Ponts ParisTech pour ses contributions à cette étude. 